\title{
Pluralities, counterparts, and groups
}

\author{
Isaac Wilhelm ${ }^{1}$
}

Accepted: 2 November 2021/Published online: 13 January 2022

(C) The Author(s), under exclusive licence to Springer Nature B.V. 2021

\begin{abstract}
I formulate a theory of groups based on pluralities and counterparts: roughly put, a group is a plurality of entities at a time. This theory comes with counterpart-theoretic semantics for modal and temporal sentences about groups. So this theory of groups is akin to the stage theory of material objects: both take the items they analyze to exist at a single time, and both use counterparts to satisfy certain conditions relating to the modal properties, temporal properties, and coincidence properties of those items.
\end{abstract}

Keywords Groups · Social metaphysics · Pluralities · Counterpart theory

\section{Introduction}

Groups are, very roughly put, bunches of objects. The U.S. Supreme court is a group, for instance: it is the group of justices which comprise it. The cheerios in my cereal bowl form a group, as do the pigeons perched along an underpass.

In the philosophical literature, groups and objects are usually analyzed in very different ways. For instance, groups have been identified with sets (Effingham, 2010), realizations of structures (Ritchie, 2013), variably plural embodiments (Uzquaino, 2018), and fusions of pluralities (Wilhelm, 2020). Objects, in contrast, are usually not identified with constructions like those. Instead, objects have been identified with bundles of properties (Williams, 1953), hylomorphic combinations of properties and parts (Johnston, 2006), spacetime worms (Lewis, 1983), and stages

Isaac Wilhelm

iwilhelm@nus.edu.sg

1 National University of Singapore, 3 Arts Link, Block AS3, \#05-22, Singapore 117570,

Singapore 
(Sider, 2001). So according to the current trend, groups and objects are ontologically dissimilar: they should be analyzed differently.

In this paper, I explore an alternative to the current trend. I formulate an account of groups - call it the 'plural account'-which is quite similar to the stage theory of material objects proposed by Sider (2001). Roughly put, according to the plural account, groups are pluralities at a single time. So just as Sider takes material objects to be instantaneous stages, the plural account takes groups to be instantaneous pluralities. And just as Sider uses counterpart theory to help his account of objects avoid various problems, I use counterpart theory to help the plural account of groups avoid various problems too.

In Sect. 2, I formulate the conditions that comprise the plural account of groups. In Sect. 3, I present some potential issues for the plural account, and I show how counterpart theory can be used to overcome them. In Sect. 4, I summarize the theory of groups that combines the plural account with counterpart theory, and I make some general observations about the relationship between objects and groups.

My main goal, in what follows, is to present and explore the plural account of groups. But I have another goal as well: this paper fills a gap in the list of proposals regarding what groups are, and in so doing, contributes to completing our picture of how accounts of groups and accounts of objects relate. As will become clear, the plural account differs from another account of groups which I have proposed (Wilhelm, 2020). Basically, according to that other account-which I unwisely ${ }^{1}$ called the 'stage theory' - groups are fusions of pluralities at worlds and times. Consequently, the stage theory of groups is, put very roughly, akin to certain worm views of material objects. In addition, worm views of material objects are different from, though obviously related to, the stage theory of material objects discussed by Sider. So a natural question is: what account of groups would stand to the stage theory of groups as worm views of material objects stand to Sider's stage theory of material objects? My answer: the plural account. So by formulating and exploring the plural account of groups, this paper helps improve our understanding of the relationships between candidate accounts of groups and candidate accounts of material objects.

\section{The plural account of groups}

The plural account of groups consists of four conditions: an existence condition, a membership condition, an identity condition, and a subgroup condition. The existence condition and the membership condition rely on certain notions from plural logic: pluralities, pluralities of pluralities, pluralities of pluralities of pluralities, and so on. The identity condition, and the subgroup condition, rely on the membership condition.

Let us start with the plural notions. A plurality is, basically, a referent of a plural term. ${ }^{2}$ For instance, consider the term 'the U.S. Supreme Court justices'. Because

\footnotetext{
${ }^{1}$ Unwisely, because Sider's stage theory of material objects is more similar to my plural account of groups than to my stage theory of groups.

${ }^{2}$ For convenience, throughout this paper, I occasionally refer to pluralities by using singular terms in English.
} 
this is a plural term, its referent is a plurality: in particular, it refers to the members of the U.S. Supreme Court. Put more roughly, but perhaps more intuitively, a plurality is just a bunch of entities. The U.S. Supreme Court justices form a plurality, for instance, since they are just a bunch of justices.

Pluralities have members. In what follows, I will refer to the relation between a plurality and its members as the ' $p_{1}$-membership' relation. ${ }^{3}$ So I will say things like "Sotomayor is a $p_{1}$-member of the U.S. Supreme Court justices." More generally, I will say that $x$ is a $p_{1}$-member of $Y$ just in case $Y$ is a plurality of entities, one of which is $x$.

As argued by Boolos (1984), pluralities are not themselves entities. That is, a plurality is not a bit of ontology over and above the entities which are its members. Plural terms merely provide another way of referring to the entities that exist; plural terms do not themselves denote extra ontology, in addition to those entities. So being ontologically committed to the existence of the U.S. Supreme Court justices, for instance, is nothing over and above being ontologically committed to the existence of each judge individually.

Just as there are bunches of entities, so there are bunches of pluralities. Following Rayo (2006), call these 'super-pluralities'. A super-plurality is, basically, a referent of a super-plural term. For instance, take the term 'overlap'. Arguably, this term is super-plural, since it can take any number of plural terms as argument places. It can be used to say things like "The square things and the blue things overlap," or "The square things, the blue things, and the wooden things overlap" (Linnebo \& Nicolas, 2008, p. 193).

Put more roughly, but more intuitively, a super-plurality is a bunch of bunches of entities. The faculty committees at a local college form a super-plurality, for instance. So do all the Supreme Courts of all the countries of the world.

As shown by Rayo (2006), these notions can be iterated indefinitely. There are super-super-pluralities, super-super-super-pluralities, and so on, up through an unending hierarchy of higher-order bunches. Super-pluralities are members of super-super-pluralities, which are members of super-super-super-pluralities, and so on.

There are 'cumulative' versions of all these 'super-' notions. A 'cumulative super-plurality', for instance, is a bunch consisting of both pluralities and entities. So whereas a super-plurality only contains pluralities as members, the members of a cumulative super-plurality may be both pluralities and entities. Similarly, a 'cumulative super-super-plurality' is a bunch consisting of entities, pluralities, and cumulative super-pluralities. And so on.

Now for some terminology. Let a '1-plurality' be a plurality. Then say that for each natural number $n$ greater than one, an ' $n$-plurality' is a cumulative super-...super-plurality, where the term 'super' is repeated $n-1$ times. Finally, say that $x$ is a ' $p_{n}$-member' of $Y$ just in case $Y$ is an $n$-plurality which contains $x$.

For example, consider the faculty committees at a local college. The hiring committee is a $p_{2}$-member of those faculty committees, since those faculty

\footnotetext{
3 The reason for the subscript will become clear shortly.
} 
committees form a 2-plurality which contains the hiring committee. Or consider the 2-plurality - call it the 'coalition' - consisting of all faculty committees and also all individuals on the college's faculty. Both the hiring committee and a professor at the college-professor Teri, say-are $p_{2}$-members of the coalition.

Just as 1-pluralities are not themselves entities, 2-pluralities are not themselves 1-pluralities (or entities). That is, a 2-plurality is not a new bit of ontology, over and above the 1-pluralities and entities which are its members. Similarly for all $n$ pluralities, where $n$ is greater than two.

It is controversial whether super-plural terms, super-super-plural terms, and so on, are intelligible, sensible, and meaningful linguistic expressions. For instance, according to Uzquiano, there is no such phenomenon as super-plural-or as Uzquiano calls it, 'plurally plural'-reference (2004, p. 146). McKay agrees (2006, p. 46), as does Lewis (1991, pp. 70-71). Linnebo, however, does not: the standard reasons for thinking that plural locutions are meaningful, Linnebo argues, motivate thinking that super-plural locutions, super-super-plural locutions, and so on, are meaningful too (2003, p. 88). Oliver and Smiley also countenance these sorts of locutions (2005, pp. 1062-1063).

In this paper, I follow Rayo, Linnebo, and others, in assuming that super-plural locutions, super-super-plural locutions, and so on, are meaningful. It may well be the case, of course, that English does not contain super-plural terms. ${ }^{4}$ Nevertheless, other languages - such as the formal languages developed in detail by Rayo (2006) - do. And for my purposes here, that is enough. It is enough to assume, in other words, that those other languages are intelligible, sensible, and meaningful. For strictly speaking, my theory of groups should not be expressed using a natural language, like English. Rather, it should be expressed using a formal, metaphysically rigorous language which contains cumulative super-plural terms, cumulative super-super-plural terms, and so on.

Now for the existence condition. Basically, it says that groups are $n$-pluralities at particular times.

\section{EXISTENCE}

For each natural number $n$ and each time $t$, every candidate $n$-plurality-that is, every candidate combination of entities, 1-pluralities, and ... and $(n-1)$ pluralities-exists at $t^{5} X_{t}$ is a group if and only if $X_{t}$ is one of these $n$ pluralities. ${ }^{6}$

In other words, a group is a bunch-of entities, 1-pluralities, or whatever-at a time.

\footnotetext{
${ }^{4}$ Rayo, for instance, claims something like this (2006). Linnebo and Nicolas, on the other hand, claim that some English terms are super-plurals (2008).

5 More precisely, for each $n$ and each $t$, I endorse a standard universal comprehension principle for $n$ pluralities at $t$.

${ }^{6}$ A straightforward generalization of this condition allows for groups whose members are drawn from arbitrarily high up in the hierarchy of $n$-pluralities.
} 
For example, consider the U.S. Supreme Court justices at the present moment. Those justices form a 1-plurality, since they are a bunch of entities. Let $S_{p}$ be that 1plurality at the present moment. Then Existence implies that $S_{p}$ is a group.

Note that according to ExISTENCE, any bunch-of entities, 1-pluralities, 2pluralities, or whatever-is a group of some sort. The 1-plurality consisting of all the $p_{1}$-members of $S_{p}$ and also all the cheerios in my cereal bowl is a group, for example. Here is another group: the 1-plurality consisting of this trout and that turkey at the present time; denote this group by ' $T_{p}$ '. So ExISTENCE implies that there are many, many groups. In particular, there are as many groups as time-slices of $n$ pluralities.

I take this to be a feature of Existence, not a bug. For it implies that some arbitrary, gerrymandered collections of entities form groups. And as I argue in other work, accounts of groups should have that implication (Wilhelm, 2020). Roughly put, the reason is this: there is no context-independent, worldly, ontological boundary between (i) those bunches which we intuitively classify as groups, and (ii) those bunches which we intuitively classify as non-groups. Pretty much any bunch of objects can seem like a group, if the context is set up right. So accounts of groups should allow that pretty much any bunch of objects is, indeed, a group. And so a good account of groups should imply that bunches like $T_{p}$ are groups. ExISTENCE does, and that is a reason to endorse it.

Because of all this, EXISTENCE is in tension with the following 'Goldilocks Constraint', endorsed by Ritchie: an ontology of social groups should include social groups that are common sensical and that figure in explanations and should not overgenerate social groups (2020, p. 403). EXISTENCE does not outright contradict the Goldilocks Constraint, of course: ExISTENCE provides an account of all groups, social and non-social; the Goldilocks Constraint concerns social groups only. So EXISTENCE is perfectly compatible with the claim that of the many groups which exist, only the common sensical, explanatory ones are social. But ExISTENCE and the Goldilocks Constraint do not sit well with one another. ExISTENCE is very permissive about what groups there are; the Goldilocks Constraint is not.

I like that EXISTENCE is in tension with the Goldilocks Constraint. For I think that the Goldilocks Constraint is probably worth rejecting. ${ }^{7}$ There were times when social groups that are now considered common sensical and explanatory-for instance, groups consisting of people with certain mental disabilities or certain nonbinary genders-were considered neither common sensical nor explanatory. Those groups still existed, at those earlier times; they were just overlooked.

More generally, the problem with the Goldilocks Constraint is its extreme sensitivity to intuitions about what does and does not exist. There are many more groups, I think, than our intuitions initially suggest. There is the group consisting of all and only blue-eyed people living, right now, in New Hampshire or Nevada. There is the group consisting of all and only the sailors-currently alive-who were born on odd-numbered days. Initially, of course, these might not strike us as groups;

\footnotetext{
7 See Epstein (2019, p. 4914) for more reasons to worry about constraints which place limitations on what groups there are.
} 
but that is just because our intuitions about groups tend to be focused on a fairly narrow range of examples. So metaphysical accounts of social groups ought to be as permissive as possible regarding what groups there are.

A good account of groups should imply that groups of groups exist (Uzquiano, 2004, p. 145). ExISTENCE does just that. To see why, let $s$ be the start of the year 2020. Consider the faculty committees at a local college at time $s$. Each of those committees is a 1-plurality: for each committee consists of a bunch of faculty, and faculty are entities. So according to ExISTENCE, at time $s$, each of those 1-pluralities is a group. Altogether, those faculty committees form a 2-plurality. So according to Existence, at time $s$, that 2-plurality is a group; denote it by ' $F_{s}$ '. And so $F_{s}$ is a group of groups: it is the group of faculty committees. ${ }^{8}$

EXISTENCE might seem to face some serious problems, however. For instance, EXISTENCE might seem to imply that groups cannot change their members. After all, $n$-pluralities with different members are non-identical, and so-it might seemgroups with different members are non-identical too.

Before addressing problems like this, I need to present the rest of the account of groups. For the solutions to these problems rely on conditions to come. So let me postpone addressing these problems until Sect. 3 .

Now for the group membership condition. Basically, it says that group membership is just a generalization of plural membership.

\section{Group Membership}

Let $t$ be a time, and let $X_{t}$ be a group. Then $e$ is a member of $X_{t}$ if and only if for some $n, e$ is a $p_{n}$-member of $X_{t}$.

For example, Sotomayor is a member of the present U.S. Supreme Court because Sotomayor is a $p_{1}$-member of the 1-plurality $S_{p}$.

Group Membership satisfies the following criterion that accounts of group membership ought to satisfy: those accounts should imply that the group membership relation is not transitive (Uzquiano, 2004, pp. 136-137; Effingham, 2010, p. 255). ${ }^{9}$ To see why accounts of group membership should allow for transitivity violations, consider the group of all faculty committees at a local college. Suppose that Teri is on the hiring committee. Then the following holds.

(1) Teri is a member of the hiring committee.

(2) The hiring committee is a member of the group of all faculty committees.

If group membership were transitive, then (1) and (2) would imply the following.

\footnotetext{
${ }^{8}$ Note that if ExISTENCE were formulated using only pluralities, then - as observed by Uzquiano (2004, p. 146) - there would not be any groups of groups; and that is why I formulated ExISTENCE using the more general notion of an $n$-plurality instead. To see the issue, consider the following 'bare plurality' account: $X_{t}$ is a group just in case $X_{t}$ is a plurality of entities at time $t$. The bare plurality account implies that if there is a group of groups, there there is a plurality of pluralities. But there are no pluralities like that: in plural logic, pluralities are not members of other pluralities; only entities are members of pluralities. So the bare plurality account does not imply that groups of groups exist. And that, of course, is problematic.

9 That transitivity principle — which accounts of group membership should falsify - is as follows: for all $x, y$, and $z$, if $x$ is a member of $y$ and $y$ is a member of $z$, then $x$ is a member of $z$.
} 
(3) Teri is a member of the group of all faculty committees.

But while (1) and (2) are true, (3) is false. Since Teri is a person, rather than a faculty committee, the group of all faculty committees does not contain Teri as a member.

Group Membership gets all that right. To see why, let $H_{s}$ be the 1-plurality of all professors on the hiring committee at time $s$, and suppose that Teri is a $p_{1}$-member of $H_{s}$. Then by Group Membership, Teri is a member of $H_{s}$; that is, (1) is true. Recall that $F_{s}$ is the group of all faculty committees at time $s$. Since $H_{s}$ is a $p_{2}$-member of $F_{s}$, Group Membership implies that $H_{s}$ is a member of $F_{s}$; in other words, (2) is true. But Teri is not a $p_{2}$-member of $F_{s}$, because $F_{s}$ only contains faculty committees as $p_{2}$-members. So by Group Membership, Teri is not a member of $F_{s}$; that is, (3) is false.

Group Membership helps capture an important distinction between groups and objects. The distinction concerns membership: while groups typically have members, objects typically do not. ${ }^{10}$ Group MEMBERSHIP says what it takes for something to be a member of a group: the members of a group are just its $p_{n^{-}}$ members. Objects, of course, do not satisfy that condition; hence, they are-in that sense-memberless.

In order to imply that empty groups exist, ExISTENCE and GRoup MEMBERSHIP must be supplemented with the assumption that there are empty pluralities. That assumption is often denied: see (Linnebo, 2003, p. 74; Rayo, 2006, pp. 230-231). But it has been endorsed as well: see (Linnebo, 2013, p. 210; Wilhelm, 2020, p. 667). So for the purposes of accommodating the existence of empty groups, I think that it is worth endorsing this controversial assumption. In what follows, I assume that empty pluralities - and therefore, empty groups-exist.

Here is the identity condition for groups. Basically, it says that groups are identical just in case they have exactly the same members at the same time.

\section{IDENTITY}

Let $t_{1}$ and $t_{2}$ be times. Let $X_{t_{1}}$ be a group which exists at $t_{1}$, and let $Y_{t_{2}}$ be a group which exists at $t_{2}$. Then $X_{t_{1}}$ is identical to $Y_{t_{2}}$ if and only if

1. $t_{1}$ is $t_{2}$,

2. every member of $X_{t_{1}}$ is a member of $Y_{t_{2}}$, and

3. every member of $Y_{t_{2}}$ is a member of $X_{t_{1}}$.

For example, IDENTITY implies that the hiring committee $H_{s}$ is identical to itself. For $s$ is $s$, and every member of $H_{s}$ is a member of $H_{s}$.

EXISTENCE and IDENTITY are analogous to the stage theory of material objects (Sider, 2001). According to the stage theory, material objects are instantaneous entities; that is, entities confined to a single time. Existence and IDENTITy say similarly for groups. According to ExISTENCE, groups are instantaneous $n$-pluralities;

\footnotetext{
${ }^{10}$ Some objects do: for instance, sets. But most objects-electrons, numbers, people, and so on-have no members.
} 
that is, groups are $n$-pluralities confined to a single time. And according to IDENTITY, a plurality at one time is distinct from a plurality at another time.

Here is an extremely attractive feature of ExISTENCE and IDENTITY: they avoid a well-known paradox. ${ }^{11}$ The paradox threatens most any account which takes groups to be objects formed out of, and individuated by, pluralities. For most of those accounts accept the following two postulates. First, for every 1-plurality $X$, there exists an object $g(X)$; these objects are the groups, according to these accounts. Second, for all 1-pluralities $X$ and $Y$, if $X$ and $Y$ are distinct-that is, if $X$ and $Y$ do not have exactly the same $p_{1}$-members-then $g(X)$ and $g(Y)$ are distinct as well. These two postulates, along with standard principles of plural logic, lead to a contradiction. $^{12}$ For they contradict a plural version of Cantor's theorem. ${ }^{13}$

Existence and Identity avoid this paradox. For Existence and Identity imply neither the first postulate nor the second. In fact, given ExISTENCE and IDENTITY, those two postulates are quite misguided. For those two postulates are only attractive insofar as they can be used to identify groups-formed out of pluralitieswith objects. And according to Existence, that identification does not hold: groups are pluralities. So EXISTENCE and IDENTITY are perfectly compatible with the denial of the postulates, and therefore, with the plural version of Cantor's theorem.

Finally, here is the subgroup condition. Basically, it says that one group is a subgroup of another just in case each member of the former is a member of the latter.

\section{SuBGROUP}

Let $t_{1}$ and $t_{2}$ be times. Let $X_{t_{1}}$ be a group which exists at $t_{1}$, and let $Y_{t_{2}}$ be a group which exists at $t_{2}$. Then $X_{t_{1}}$ is a subgroup of $Y_{t_{2}}$ if and only if

1. $t_{1}$ is $t_{2}$, and

2. every member of $X_{t_{1}}$ is a member of $Y_{t_{2}}$.

For example, consider the hiring committee $H_{s}$ once more. Suppose that professor Skyler, in addition to Teri, is on the hiring committee. And suppose that Teri and Skyler comprise a special subcommittee of $H_{s}$; call it $S_{s}$. Then Subgroup implies that $S_{s}$ is a subgroup of $H_{s}$. For $s$ is $s$, and $H_{s}$ contains the members of $S_{s}$-namely, Teri and Skyler-as members. ${ }^{14}$

\footnotetext{
11 Thanks to an anonymous referee for pointing this out.

12 In rough outline, here is how. The two postulates say, basically, that there exists an injection $g$ from pluralities to objects. But the existence of that injection is inconsistent with the logic of plurals. For suppose that this injection $g$ exists. Then by a principle of plural comprehension, there exists a plurality $Z$ such that $z$ is a $p_{1}$-member of $Z$ if and only if there is an $X$ such that (i) $z=g(X)$, and (ii) $z$ is not a $p_{1^{-}}$ member of $X$; think of $Z$ as the plurality of all groups $g(X)$ that are not contained in their corresponding plurality $X$. Now consider $g(Z)$. Either $g(Z)$ is a $p_{1}$-member of $Z$, or $g(Z)$ is not a $p_{1}$-member of $Z$. But in each case, as a simple proof shows, contradiction results. So there is no such function as $g$.

13 For more discussion of this paradox, see (Landman, 1989). The paradox is also discussed in (Uzquiano, 2018, p. 428).

14 Many philosophers, myself included, often refer to conditions like SubGRoup as parthood conditions (Uzquiano, 2018, p. 448; Wilhelm, 2020, p. 670). I now prefer to describe conditions like SubGrouP as subgroup conditions instead: it is more natural to talk about subgroups of groups than to talk about parts of groups.
} 
Existence, Group Membership, Identity, and Subgroup, form what I have been calling the 'plural account' of the metaphysics of groups. According to the plural account, groups are just generalized pluralities: bunches of entities, bunches of entities and pluralities, and so on. ${ }^{15}$ Group membership is just a generalization of plural membership. Group identity is just a generalization of plural identity. And the notion of a subgroup is just a generalization of the notion of a subplurality. ${ }^{16}$

\section{Modality, time, and counterpart theory}

In this section, I discuss some problems which might seem to arise for the plural account. In Sect. 3.1, I describe those problems. In Sect. 3.2, I explain how those problems can be solved: the solutions appeal to counterpart theory.

\subsection{Three problems}

There are three problems which the plural account might seem to face. First, it might seem to face a problem concerning the modal properties of groups. Second, it might seem to face a problem concerning the temporal properties of groups. Third, it might seem to face a problem concerning groups that always coincide. Let us examine these problems in detail.

First, the 'modal problem': the plural account might seem to imply that it is impossible for groups to be different from how they actually are. For example, consider the group of U.S. Supreme Court justices. Intuitively, that group could have had different members. For instance, intuitively, Merrick Garland ${ }^{17}$ could have been a member of it. But the plural account might seem to contradict this. To see why, take any group $G_{t}$-at any time $t$-which contains Garland as a member. Garland is not a member of $S_{p}$; that is, Garland is not a member of the group consisting of all U.S. Supreme Court justices at the present time. So by IDENTITY, $G_{t}$ and $S_{p}$ are distinct. And so the plural account might seem to imply that $S_{p}$ could not

\footnotetext{
15 This is one of the main differences between the plural account and the account of social groups proposed by Faller (2021). Both accounts take groups to be stages, in some sense or other; and to avoid various problems, both appeal to counterpart theory. But whereas my account takes the stages to be $n$ pluralities at times, Faller's account does not. Moreover, Faller's account focuses on social groups in particular, and takes those groups to obey the Goldilocks Constraint. Not so for the plural account.

16 The plural account respects the explanatory role which groups play in many scientific theories. For scientific theories of groups, according to the plural account, are basically just scientific theories of pluralities at times. And scientific theories of pluralities at times, when true, are extremely explanatory. Take economic theories that use the group of U.S. stockholders to explain the recession caused by Covid19 , or child psychology theories that use the group of U.S. teenagers to explain nation-wide patterns of young adult behavior. These theories explain a wide variety of phenomena. And they do so, according to the plural account, by invoking pluralities at particular times - that is, by invoking groups.

17 Garland was one of Obama's nominees to the U.S. Supreme Court. He was never confirmed as a Supreme Court justice.
} 
have been $G_{t}{ }^{18}$ It might seem impossible, in short, for any group to be other than how it actually is.

Second, there is a 'temporal problem': the plural account might seem to imply that groups do not change over time. For example, consider once more the group of U.S. Supreme Court justices. From the year 2000 to today, that group has changed its members. For instance, Scalia was a member of that group in 2000, but he is not a member of it now. The plural account, however, might seem to contradict this. To see why, let $S_{2000}$ be the 1-plurality consisting of all U.S. Supreme Court justices at the start of the year 2000. By Existence, $S_{2000}$ is a group. By Group Membership, Scalia is a member of $S_{2000}$. Scalia is not a member of $S_{p}$, however. So by IDENTITY, $S_{2000}$ and $S_{p}$ are distinct. And so the plural account might seem to imply that no group changed its members, from the start of 2000 to the present moment. No one thing — no one group—seems to have changed.

Third, there is a 'coincidence problem': the plural account might seem to violate the intuition that distinct groups can sometimes coincide. For instance, consider a Dungeons and Dragons group which meets at a local college. And consider that college's cycling group. Let $D_{p}$ be the Dungeons and Dragons group at the present time, and let $C_{p}$ be the cycling group at the present time. Suppose, furthermore, that these groups have exactly the same members: the members of the Dungeons and Dragons group are all and only the members of the cycling group. Then IDENTITY implies that these groups are one and the same. But that might seem problematic. For intuitively, the Dungeons and Dragons group is distinct from the cycling group.

To summarize: there are three problems which the plural account might seem to face. First, it might seem to imply that groups cannot be different from how they actually are. Second, it might seem to imply that groups cannot change over time. Third, it might seem to imply that distinct groups cannot coincide.

\subsection{Solutions}

These three problems are not unique to the plural account of groups. Entirely analogous problems arise for the stage theory of material objects. And in the case of material objects, those problems can be solved. Proponents of the stage theory often solve those problems by adopting counterpart-theoretic semantics for modal and temporal sentences about objects (Sider, 2001).

An analogous strategy can be used to solve the three problems facing the plural account. In this section, I explain how. That is, I show that by supplementing the

\footnotetext{
18 This follows from the plural account along with standard assumptions about the necessity of identity (Kripke, 1980).
} 
plural account with counterpart-theoretic semantics for modal and temporal sentences about groups, the three problems can be avoided. ${ }^{19}$

By way of preparation, it is worth reviewing the counterpart-theoretic approach to the semantics of modal sentences. ${ }^{20}$ According to what I will call 'modal counterpart theory', modal sentences about groups in the actual world are true because of similar groups - call them 'modal counterpart' groups - in other worlds. For example, consider the sentence "Garland could have been a member of the U.S. Supreme Court." According to modal counterpart theory, this sentence is true because there is a possible world in which the following holds: a modal counterpart of Garland is a member of a modal counterpart of the U.S. Supreme Court.

The modal counterpart relation-the relation, that is, which obtains between an actual-world item and its modal counterparts-is determined by similarity. In particular, if $x$ is an entity in the actual world, then a modal counterpart of $x$ in possible world $w$ is an entity in $w$ which is at least as similar to $x$ as each other entity in $w$ is. And if $X_{t}$ is a group in the actual world, then a modal counterpart of $X_{t}$ in possible world $w$ is a group in $w$ which is at least as similar to $X_{t}$ as each other group in $w$ is. The similarity relation varies from context to context: two groups might be extremely similar in one context, but not in another. For example, consider a possible world in which one 1-plurality of justices decided on the case Ramos $v$. Louisiana while another, distinct 1-plurality of justices decided on the case Barton $v$. Barr. Depending on the context, the first group or the second-or both, or neither-may be sufficiently similar to the group $S_{p}$ of present U.S. Supreme Court justices to be one of its modal counterparts.

Think of this modal counterpart relation as having two parts; or rather, two subrelations. One relates entities in a world to other entities-their modal counterparts-in other worlds. Call this the 'entity modal counterpart relation'. The other relates groups in a world to other groups - their modal counterparts-in other worlds. Call this the 'group modal counterpart relation'.

There are at least two ways of understanding the relationship between the group modal counterpart relation and the entity modal counterpart relation. For starters, one might analyze the former in terms of the latter. In particular, one might adopt the following principle: group $Y_{t}$ in world $w_{Y_{t}}$ is a modal counterpart of group $X_{t}$ in world $w_{X_{t}}$ if and only if

\footnotetext{
19 The stage theory of material objects faces other problems too, and versions of those problems arise for the plural account of groups. According to Balashov, for instance, the stage theory of material objects conflicts with special relativity (2002); Balashov's argument, if correct, could be used to show that the plural account of groups conflicts with special relativity as well. Ultimately, I think that the plural account of groups, when supplemented with the counterpart-theoretic semantics to come, supports good responses to objections like this one. But for lack of space, I will not discuss that here. Instead, I simply wish to show that those who like the stage theory of material objects should also like the combination of (i) the plural account of groups, and (ii) the forthcoming account of the semantics of temporal and modal sentences about groups. For that combination solves many problems relating to groups, and it does so without introducing any new problems with which stage theorists are unfamiliar.

${ }^{20}$ For a detailed discussion of counterpart theory, see (Lewis, 1986).
} 
(i) each member of $Y_{t}$ is a modal counterpart of some member of $X_{t}$, and

(ii) each member of $X_{t}$ is a modal counterpart of some member of $Y_{t}{ }^{21}$

Alternatively, one might claim that the group modal counterpart relation cannot be analyzed in terms of the entity modal counterpart relation. Neither relation, one might claim, reduces to the other: they are separate, non-interdefinable relations in the semantics to come.

I prefer the latter option. For I think that one group may be a modal counterpart of another even if, for whatever reason, some member of the former has no modal counterpart in the latter. As an example, consider a possible world $w$ that, in most respects, is extremely similar to the actual world. But suppose that in $w$, the U.S. Supreme Court at the present time — call it ' $S_{w, p}$ ' — has no chief justice; perhaps the U.S. Supreme Court in $w$ has only ever had eight justices, none of whom outrank any of the others. Then arguably, $S_{w, p}$ does not contain a modal counterpart of the chief justice of $S_{p}$ in the actual world. Nevertheless, $S_{w, p}$ and $S_{p}$ may still be modal counterparts.

Now for the account of the truth conditions of modal sentences about groups. Let $t$ be a time, let $X_{t}$ be a group at $t$, let $c$ be a context, and let $\phi$ be a sentence. As usual, suppose that $c$ determines the counterparts of $X_{t}$ in various worlds; call these the 'modal $c$ counterparts' of $X_{t}$. Then the truth conditions for modal sentences are as follows.

\section{Possibility}

" $X_{t}$ could have been such that $\phi "$ is true at $t$ in $c$ if and only if in some possible world, a modal $c$-counterpart of $X_{t}$ is such that $\phi$.

In other words, to evaluate the truth value of the modal sentence "Such-and-such group could have been thus-and-so," do the following. First, for each world, determine which groups-if any-are modal counterparts of the such-and-such group in question. Second, check whether at least one of those modal counterparts, in at least one of those worlds, is thus-and-so. If so, then the modal sentence is true. If not, then the modal sentence is false.

For example, consider the sentence below.

(Gar) The U.S. Supreme Court could have had Garland as a member.

Intuitively, (Gar) is true. PossiBiLity gets that right. To see why, note that there are many possible worlds which contain a modal $c$-counterpart of the U.S. Supreme Court. At least one of those modal $c$-counterpart groups contains Garland-or rather, a counterpart of Garland-as a member. So PossiBILITY implies that (Gar) holds.

PossibiLITy can be used to solve the modal problem. For when combined with the plural account, PossibiLity implies that many groups could indeed have been otherwise. The present U.S. Supreme Court-that is, $S_{p}$-could indeed have

\footnotetext{
${ }^{21}$ A tedious exercise for the reader: show, by double induction, that this condition really does reduce each claim of the form "group $Y_{t}$ is a modal counterpart of group $X_{t}$ " - where $Y_{t}$ is an $n$-plurality for some $n$, and $X_{t}$ is an $m$-plurality for some $m$-to a complicated series of claims which only mention entities and 1-pluralities to which they belong.
} 
contained Garland as a member; that is what (Gar) says. So the plural account does not imply that it is impossible for groups to be different from how they actually are. When combined with the proper account of the truth conditions of modal sentences about groups - namely, PossibiLity - the plural account respects the fact that many groups could have been different.

Just as there is a counterpart-theoretic approach to the semantics of modal sentences, so there is a counterpart-theoretic approach to the semantics of temporal sentences. ${ }^{22}$ According to what I will call 'temporal counterpart theory', temporal sentences about groups are true at the present time because of similar groups-call them 'temporal counterpart' groups-at other times. For example, consider the sentence "Scalia was a member of the U.S. Supreme Court." According to temporal counterpart theory, this sentence is true because there is a past time at which Scalia is a member of a temporal counterpart of the present U.S. Supreme Court.

Just as with the modal counterpart relation, the temporal counterpart relationthe relation, that is, which obtains between an item and its temporal counterpartsis determined by similarity. In particular, if $X_{t}$ is a group at time $t$, then a temporal counterpart of $X_{t}$ at time $t^{\prime}$ is a group $Y_{t^{\prime}}$ which is at least as similar to $X_{t}$ as each other group at $t^{\prime}$ is. The similarity relation, as always, varies from context to context: two groups might be extremely similar in one context, but not in another. ${ }^{23}$

Now for the account of the truth conditions of temporal sentences about groups. Let $t$ be a time, let $X_{t}$ be a group at $t$, let $c$ be a context, and let $\phi$ be a sentence. As usual, suppose that $c$ determines the temporal counterparts of $X_{t}$ at various times; call these the 'temporal $c$-counterparts' of $X_{t}$. Then the truth conditions for temporal sentences about the past, present, and future, are as follows.

PAST

" $X_{t}$ was such that $\phi$ " is true at $t$ in $c$ if and only if at some time $t^{\prime}$ earlier than $t$, a temporal $c$-counterpart of $X_{t}$ is such that $\phi .^{24}$

PRESENT

" $X_{t}$ is now such that $\phi$ " is true at $t$ in $c$ if and only if at $t$, a temporal $c$ counterpart of $X_{t}$ is such that $\phi .^{25}$

\footnotetext{
${ }^{22}$ For details, see (Sider, 2001).

23 Just as with the modal counterpart relation, the temporal counterpart relation can be thought of as having two parts; that is, two sub-relations. One relates entities, while the other relates groups. And just as with the modal counterpart relation, instances of the temporal counterpart relation that obtain between groups should not be reductively analyzed in terms of instances of the temporal counterpart relation that obtain between entities only.

24 Note that the 'is' in the metalanguage expression 'is such that $\phi$ ' - on the right side of the biconditional—should be read tenselessly. Following Sider (2001, p. 193), I formulate my account of the truth conditions of the tensed language in question here-namely, English-by using a tenseless metalanguage.

25 I use the word 'now' to emphasize that the 'is' in " $X_{t}$ is now such that $\phi$ " should be read as tensed. As mentioned in footnote 24 , the 'is' in the metalanguage expression 'is such that $\phi$ ' - on the right side of the biconditional—should be read tenselessly.
} 


\section{FUTURE}

" $X_{t}$ will be such that $\phi$ " is true at $t$ in $c$ if and only if at some time $t^{\prime}$ later than

$t$, a temporal $c$-counterpart of $X_{t}$ is such that $\phi$.

In other words, to evaluate the truth value of a temporal sentence like "Such-andsuch group was thus-and-so," do the following. First, for each earlier time, determine which groups-if any-are temporal counterparts of the such-and-such group in question. Second, check whether at least one of those temporal counterparts is thus-and-so. If so, then the temporal sentence is true. If not, then the temporal sentence is false. Likewise for temporal sentences about the present, and for temporal sentences about the future too.

As an example, consider the sentence below.

(Sca) The U.S. Supreme Court had Scalia as a member.

Intuitively, (Sca) is true. PAST gets that right. To see why, note that there are many past times which contain a temporal $c$-counterpart of the U.S. Supreme Court. Some-indeed, many-of those temporal $c$-counterpart groups contain Scalia as a member. So PAST implies that (Sca) holds.

Past, Present, and Future can be used to solve the temporal problem. For when combined with the plural account, PAst, Present, and Future imply that many groups do indeed change over time. Scalia really was a member of the U.S. Supreme Court, for instance; that is what (Sca) says. So the plural account does not imply that groups never change. When combined with the proper account of the truth conditions of temporal sentences about groups-namely, PAst, Present, and FUTURE- the plural account respects the fact that many groups change over time.

Now for the solution to the coincidence problem. As will become clear, the solution is somewhat involved. Basically, there are two reasons why. First, the solution appeals to an additional aspect of counterpart theory, one which I have not yet introduced. Second, the additional aspect of counterpart theory initially appears to contradict the plural account, since IDENTITY appears to be inconsistent with it. So in what follows, I explain the additional aspect of counterpart theory which the solution employs, I illustrate that aspect of counterpart theory with an example, and then I explain why it does not contradict IDENTITY.

The additional aspect of counterpart theory is as follows: different ways of referring to a group can, roughly put, make multiple similarity relations salient (Kocurek, 2018; Lewis, 1973). Those similarity relations, in turn, can generate multiple modal counterpart relations and multiple temporal counterpart relations: one modal counterpart relation, and one temporal counterpart relation, for each contextually salient relation of similarity. As a result, groups may have multiple modal counterparts at the same worlds, and groups may have multiple temporal counterparts at the same times. And so the truth conditions of identity sentences about coincident groups can vary, depending on (i) the terms used to denote those coincident groups, and (ii) whether or not those terms generate different contextually salient similarity relations.

Here are the details. Let $t$ be a time. Let ' $a$ ' and ' $b$ ' be terms that, in the actual world, denote the same group: in particular, ' $a$ ' and ' $b$ ' denote the same $n$-plurality at the same time $t$. Let $c$ be a context. Suppose that $c$ determines two similarity 
relations: one associated with the term ' $a$ ' and another associated with the term ' $b$ '. Each similarity relation generates a different modal counterpart relation: a modal $c_{a^{-}}$ counterpart relation, and a modal $c_{b}$-counterpart relation. ${ }^{26}$ Then the truth conditions for identity sentences are as follows.

SAME

The sentence " $a$ is $b$ " is true at $t$ in $c$ if and only if at $t$,

1. every modal $c_{a}$-counterpart of $a$ is a modal $c_{b}$-counterpart of $b$, and

2. every modal $c_{b}$-counterpart of $b$ is a modal $c_{a}$-counterpart of $a$.

The truth conditions for distinctness sentences are similar.

\section{DisTINCT}

The sentence " $a$ is distinct from $b$ " is true at $t$ in $c$ if and only if at $t$,

1. some modal $c_{a}$-counterpart of $a$ is not a modal $c_{b}$-counterpart of $b$, or

2. some modal $c_{b}$-counterpart of $b$ is not a modal $c_{a}$-counterpart of $a$.

Similar truth conditions can be formulated for other sentences that feature the terms ' $a$ ' and ' $b$ '. 27

For example, recall the Dungeons and Dragons group $D_{p}$ and the cycling group $C_{p}$ from Sect. 3.1. $D_{p}$ and $C_{p}$ have exactly the same members at the present time $p$, and so by IDENTITy, they are identical. Nevertheless, intuitively, $D_{p}$ and $C_{p}$ are distinct in the present context $c$. That is, in $c$, the following English-language sentence seems true.

(Dis) The Dungeons and Dragons group is distinct from the cycling group.

Distinct gets that right. To see why, note that in $c$, the two expressions 'the Dungeons and Dragons group' and 'the cycling group' make different features of the group in question salient. For instance, people who play Dungeons and Dragons are often fantasy game enthusiasts, and they are-stereotypically-often somewhat awkward. Cyclists, on the other hand, are often exercise enthusiasts, and they often like spending lots of time outside. So according to the counterpart relation most naturally associated with the expression 'the Dungeons and Dragons group' - call it the 'modal $c_{d}$-counterpart relation' - a modal counterpart of that group is a group whose members are fantasy game enthusiasts, socially awkward, and so on. And according to the counterpart relation most naturally associated with the expression 'the cycling group' - call it the 'modal $c_{c}$-counterpart relation' - a modal counterpart of that group is a group whose members are outdoor enthusiasts, often outside, and so on. Plausibly, in some possible world, a modal $c_{d}$-counterpart of the Dungeons and Dragons group is not a modal $c_{c}$-counterpart of the cycling group: the

\footnotetext{
${ }^{26}$ In what follows, I focus on modal counterpart relations. Temporal counterpart relations could be used instead.

27 To do so, replace all instances of ' $a$ ' and ' $b$ ', in the sentence in question, by variables. An open, quasiEnglish sentence will result. Then say that the original sentence is true just in case every modal $c_{a^{-}}$ counterpart of $a$ and every modal $c_{b}$-counterpart of $b$ satisfies the corresponding open sentence, when those counterparts are assigned to the appropriate variables.
} 
members of the former group do not enjoy outdoor activities, say; and the members of the latter group do not enjoy fantasy games. So Distinct implies that in the present context $c$, "The Dungeons and Dragons group is distinct from the cycling group" is true. That is, in $c$, Distinct implies that (Dis) holds.

This might seem to contradict IDENTITY. According to Distinct, the sentence (Dis) is true in $c$. But recall that 'the Dungeons and Dragons group' refers to $D_{p}$, while 'the cycling group' refers to $C_{p}$. And IDENTITY implies that $D_{p}$ and $C_{p}$ are identical, since they have the same members and they are indexed to the same time. So how could $D_{p}$ and $C_{p}$ be identical, given that (Dis) is true in context $c$ ?

The answer: IDENTITY and DistinCT play importantly different, largely orthogonal roles in the overall theory of groups being explored here. IDENTITY helps to characterize the metaphysics of groups. It states the conditions under which groups are identical, in the strictest, most literal, most metaphysically rigorous sense. Distinct helps to characterize the semantics of ordinary talk about groups. It states the conditions under which certain natural-language sentences-whose naturallanguage terms refer to groups-are true.

In other words, whereas IDENTITY is a condition of metaphysics, Distinct is a condition of natural-language semantics. IDENTITY has no straightforward implications for the truth conditions of natural-language expressions like 'the Dungeons and Dragons group' 'the cycling group'. And Distinct has no straightforward implications for the metaphysics of 1-pluralities like $D_{p}$ or $C_{p} \cdot{ }^{28}$ So IDENTITY and Distinct do not contradict each other: they belong to distinct regions of the overall theory of groups under consideration.

Plausibly, Distinct solves the coincidence problem. Whether or not it does, however, depends on exactly what one takes the coincidence problem to be. One might take it to be a problem concerning pre-theoretic intuitions which we express using natural language. To satisfy those pre-theoretic intuitions, expressed using sentences of English, one might claim that it suffices to give a semantics which yields the intuitively correct truth conditions for those sentences. And DisTinct does just that.

One might interpret the coincidence problem differently, however. In particular, one might take it to be a problem concerning rigorous metaphysics. So solving the problem, one might insist, involves more than just accounting for the intuitively correct truth conditions of certain natural-language sentences. In order to solve the problem, one might insist, an account of groups must imply that in the most metaphysically rigorous sense, there exist numerically distinct groups which have exactly the same members at exactly the same times. The plural account does not have this implication, and so-one might argue - that is a reason to reject it.

In response, fans of the plural account should claim that this is the wrong interpretation of the coincidence problem. The intuitions to be captured are rough, hazy, and pre-theoretic. They did not originate in the context of rigorous metaphysical theorizing. They originated in more everyday contexts: conversations with friends, the familiar practice of joining and leaving various groups, and so on. So to solve the

\footnotetext{
${ }^{28}$ Of course, while writing this paper, I used English to express both (i) the rigorous metaphysical theory of groups, and (ii) the semantic theory of ordinary talk about groups. But that is just a contingent feature of how English speakers write papers and communicate ideas.
} 
coincidence problem, an account of groups need not validate the relevant intuitions at the level of formal, rigorous metaphysics: for that is not the level with which the relevant intuitions are concerned. Rather, to solve the coincidence problem, an account of groups need only validate the relevant intuitions at the level of everyday talk about groups. Distinct does that, and so Distinct solves the coincidence problem.

An autobiographical aside: for my part, I am undecided as to whether this is, indeed, the best solution to the coincidence problem. There is certainly much to like about this counterpart-theoretic solution. But as I have argued elsewhere, there is also much to like about solutions which-like the solution supported by the stage theory of groups in (Wilhelm, 2020)_imply that in the most metaphysically rigorous sense, some numerically distinct groups have exactly the same members at exactly the same times. Ultimately, I suspect that the issue will be decided by more general considerations than I can explore here. ${ }^{29}$

There is another worry which one might raise for this solution to the coincidence problem. One might worry that this solution does not respect the following fact: coincident groups can have different causal powers. For example, suppose that at a local college, the exact same people belong to both the examination committee and the library committee. If these groups are identical, one might worry, then their causal powers must be identical too. But that is clearly wrong. The examination committee has causal powers which the library committee lacks: the examination committee can hold exams, for instance.

My response: that variation in causal powers, between the examination committee and the library committee, is compatible with those committees being one and the same. For on my preferred view, the causal powers of groups should be understood in terms of counterfactuals. And the truth conditions of those counterfactuals should be understood in terms of counterpart theory. So in particular, for the examination committee to have the causal power to hold exams just is for certain counterfactuals-like "If the examination committee had not made thus-and-so decision, then the exam would not have been held"- to be true. For the library committee to lack that causal power just is for certain counterfactuals-like "If the library committee had not made thus-and-so decision, then the exam would not have been held" - to be false. And this is all perfectly compatible with IDENTITY: this is all perfectly compatible, that is, with the examination committee and the library committee being identical. It just means that causation ultimately reduces to facts about certain counterfactuals' truth values - and those truth values depend on the terms which those counterfactuals feature.

In other work, I discuss this dependence-of counterfactuals' truth values on terms - in detail (Wilhelm, 2021). As I argue, identity facts can explain other facts.

\footnotetext{
${ }^{29}$ For instance, considerations from physics suggest that the solution supported by the stage theory of groups may be best. As I show elsewhere, worm views of material objects can be used to solve some problems for the Everett interpretation of quantum mechanics (Wilhelm, forthcoming). And that solution, I suspect, meshes better with the stage theory of groups than with the plural account of groups. But some considerations push in the opposite direction. In particular, certain theories of explanation suggest that the counterpart-theoretic solution to the coincidence problem may be better than the solution supported by the stage theory. For the counterpart-theoretic solution meshes better with my preferred account of how identity facts explain; I discuss that account below.
} 
The fact that the cycling group is identical to the Dungeons and Dragons group, for instance, explains the fact that the cycling group went campaigning on Wednesday. Ultimately, I propose the 'counteridentical account' of how identity facts explain: very roughly put, identity facts explain by figuring in true, explanatory counteridenticals (Wilhelm, 2021, p. 58) ${ }^{30}$ According to this account, counteridenticals like "If the cycling group were not the Dungeons and Dragons group, then the cycling group would not have gone campaigning on Wednesday" are both true and explanatory. And that is why the corresponding identify fact-the fact that the cycling group is indeed identical to the Dungeons and Dragons group-explains the fact that the cycling group went campaigning on Wednesday.

DisTinct combines quite nicely with the counteridentical account; and that, I think, speaks well of them both. The truth conditions for Distinct, and the counteridentical account's truth conditions for counteridenticals, posit multiple counterpart relations: one for each term in statements of identity. That posit, in turn, plays two strategic roles: it both (i) solves the coincidence problem, and also (ii) captures the explanatory capacities of identity facts about groups. Of course, Distinct and the counteridentical account need not stand or fall together: either can be endorsed without endorsing the other. But they cohere with one another quite well. And that is, I think, a significant point in their favor.

Taken together, Possibility, Past, Present, Future, Same, and Distinct, form a semantics for natural-language sentences about groups; call it the 'counterparttheoretic' account. The basic idea of the counterpart-theoretic account is that the truth values of modal sentences about groups, and temporal sentences about groups, are determined by those groups' modal and temporal counterparts. In addition, the truth values of identity sentences about groups-expressed in a natural language like English-are determined by counterparts too.

Note that the counterpart-theoretic account satisfies the following desiderata, drawn from (Epstein, 2015; Ritchie, 2013; Uzquiano, 2018).

(i) Some groups have their members contingently; so sentences like (Gar) are true.

(ii) Some groups have their members temporarily; so sentences like (Sca) are true.

(iii) Some groups exist at some but not all worlds. ${ }^{31}$

(iv) Some groups exist at some but not all times. ${ }^{32}$

(v) Distinct groups can sometimes coincide; so sentences like (Dis) are true in certain contexts.

\footnotetext{
30 A counteridentical is a counterfactual whose antecedent invokes an identity statement.

31 To see why the counterpart-theoretic account satisfies this desiderata, consider a sentence like "The U.S. Supreme Court could have failed to exist." This sentence is true, according to the counterparttheoretic account, because there are worlds which contain no modal counterparts of the U.S. Supreme Court.

32 To see why the counterpart-theoretic account satisfies this desiderata, consider a sentence like "The U.S. Supreme Court used to not exist." This sentence is true, according to the counterpart-theoretic account, because there are past times at which no temporal counterpart of the U.S. Supreme Court exists.
} 
So the counterpart-theoretic account successfully captures the intuitively correct truth conditions of various modal, temporal, and identity sentences-expressed in natural languages - about groups.

\section{The plural counterpart theory}

Let the 'plural counterpart' theory of groups be (i) the plural account of the metaphysics of groups, combined with (ii) the counterpart-theoretic account of the semantics of natural-language sentences about groups. The plural counterpart theory is a theory of group existence, group membership, group identity, subgroups, and natural-language sentences about groups that involve modality, time, and identity. So the plural counterpart theory provides a reasonably complete theory of group metaphysics and group semantics.

The plural counterpart theory of groups is similar to the stage theory of material objects. Both take the relevant items of analysis-groups and objects, respectively - to exist at a single world and a single time. And in order to avoid various problems, both adopt counterpart-theoretic semantics for modal and temporal sentences. ${ }^{33}$

According to the plural counterpart theory and the stage theory, however, there are two significant differences between groups and objects. First, objects are just singular items, whereas groups are items considered in plural. To put it another way, objects are entities taken singularly, and pluralities are entities taken plurally. Second, groups typically have members, whereas objects typically do not. Because of that, groups - but not objects-have membership conditions.

In closing, I want to make a tentative methodological point. In the philosophical literature, theories of groups have often differed - quite radically_-from theories of

\footnotetext{
33 Relatedly, it is worth making one final observation about the plural counterpart theory of groups, the stage theory of groups that I have explored elsewhere, and accounts of objects. For the reasons just given, the stage theory of material objects is akin to the plural counterpart theory. For reasons I discuss elsewhere, worm views of material objects are similar to the stage theory of groups (Wilhelm, 2020, p. 664). So altogether, I tentatively suggest the following: fans of the stage theory of material objects should endorse the plural counterpart theory of groups, while fans of worm views of material objects should endorse the stage theory of groups. These are the most natural combinations of views, for those who think that both objects and groups exist, to endorse.
} 
material objects. Nevertheless, it is worth exploring whether theories of groups and theories of objects must really be all that different. ${ }^{34}$ For as the plural counterpart theory shows, at least one reasonable theory of groups is quite similar to a standard theory of material objects: namely, the stage theory. So perhaps philosophical theories of groups should not assume, from the get-go, that groups and objects are radically different. Perhaps it is worth exploring the extent to which theories of objects might serve as a guide for formulating plausible, defensible, attractive theories of groups (Linnebo 2013).

Acknowledgements Thanks to Karen Bennett, Christian Lee, Aidan Ottoni-Wilhelm, Dee Payton, Jonathan Schaffer, Neal Tognazzini, Ryan Wasserman, and Dennis Whitcomb, for much helpful feedback and discussion.

\section{References}

Baker, L. R. (2019). Just what is social ontology? Journal of Social Ontology, 5(1), 1-12.

Balashov, Y. (2002). On Stages, Worms, and Relativity. In C. Callender (Ed.), Time, Reality \& Experience. Cambridge University Press, pp. 223-252.

Boolos, G. (1984). To be is to be a value of a variable. The Journal of Philosophy, 81(8), 430-449.

Effingham, N. (2010). The metaphysics of groups. Philosophical Studies, 149(2), 251-267.

Epstein, B. (2015). The Ant Trap. Oxford University Press.

Epstein, B. (2019). What are social groups? Their metaphysics and how to classify them. Synthese, 196(12), 4899-4932.

Faller, A. (2021). How groups persist. Synthese, 198, 7149-7163.

Hindriks, F. A. (2020). Establishments as Material rather than Immaterial Objects. Australasian Journal of Philosophy. https://doi.org/10.1080/00048402.2020.1821724

Johnston, M. (2006). Hylomorphism. The Journal of Philosophy, 103(12), 652-698.

Kocurek, A. W. (2018). Counteridenticals. The Philosophical Review, 127(3), 323-369.

Korman, D. Z. (2020). The Metaphysics of Establishments. Australasian Journal of Philosophy, 98(3), 434-448.

Kripke, S. A. (1980). Naming and Necessity. Basil Blackwell.

Landman, F. (1989). Groups, I. Linguistics and Philosophy, 12(5), 559-605.

Lewis, D. (1973). Counterfactuals. Blackwell.

Lewis, D. (1983). Survival and Identity. In Philosophical Papers. Oxford University Press, Vol. 1, pp. $55-72$.

Lewis, D. (1986). On the Plurality of Worlds. Blackwell.

Lewis, D. (1991). Parts of Classes. Blackwell.

Linnebo, Ø. (2003). Plural quantification exposed. Nô̂s, 37(1), 71-92.

Linnebo, Ø. (2013). The potential hierarchy of sets. The Review of Symbolic Logic, 6(2), 205-228.

Linnebo, Ø., \& Nicolas, D. (2008). Superplurals in English. Analysis, 68(3), 186-197.

McKay, T. J. (2006). Plural Predication. Oxford University Press.

Oliver, A., \& Smiley, T. (2005). Plural descriptions and many-valued functions. Mind, 114(456), 1039-1068.

\footnotetext{
${ }^{34}$ For recent explorations of this, see the theories of groups-and establishments, and other aspects of the social world-discussed by Baker (2019), Hindriks (2020), and Korman (2020). Those theories, like mine, challenge the assumption that approaches to the metaphysics of groups must be radically different from approaches to the metaphysics of objects. So to that extent, those theories and my theory are working towards a common goal. There is an important difference between those theories and mine, however: whereas those theories generally take groups to be special sorts of objects, mine does not. Korman argues that groups are immaterial objects, for instance (2020, p. 443); and Hindriks argues that groups are material objects whose existences can depend on decisions (2020, pp. 4-5). I have argued, in contrast, that groups are pluralities rather than objects.
} 
Rayo, A. (2006). Beyond Plurals. In A. Rayo \& G. Uzquiano (Eds.), Absolute Generality. Oxford University Press, pp. 220-254.

Ritchie, K. (2013). What are groups? Philosophical Studies, 166(2), 257-272.

Ritchie, K. (2020). Social structures and the ontology of social groups. Philosophy and Phenomenological Research, 100(2), 402-424.

Sider, T. (2001). Four-Dimensionalism. Oxford University Press.

Uzquiano, G. (2004). The supreme court and the supreme court justices: A metaphysical puzle. Nô̂s, 38(1), 135-153.

Uzquiano, G. (2018). Groups: Toward a theory of plural embodiment. The Journal of Philosophy, 115(8), $423-452$.

Wilhelm, I. (2020). The Stage Theory of Groups. Australasian Journal of Philosophy, 98(3), 661-674.

Wilhelm, I. (2021). The Counteridentical Account of Explanatory Identities. The Journal of Philosophy, $118(2), 57-78$.

Wilhelm, I. (forthcoming). Centering the Everett Interpretation. The Philosophical Quarterly.

Williams, D. C. (1953). On the elements of being: II. The Review of Metaphysics, 7(2), 171-192.

Publisher's Note Springer Nature remains neutral with regard to jurisdictional claims in published maps and institutional affiliations. 